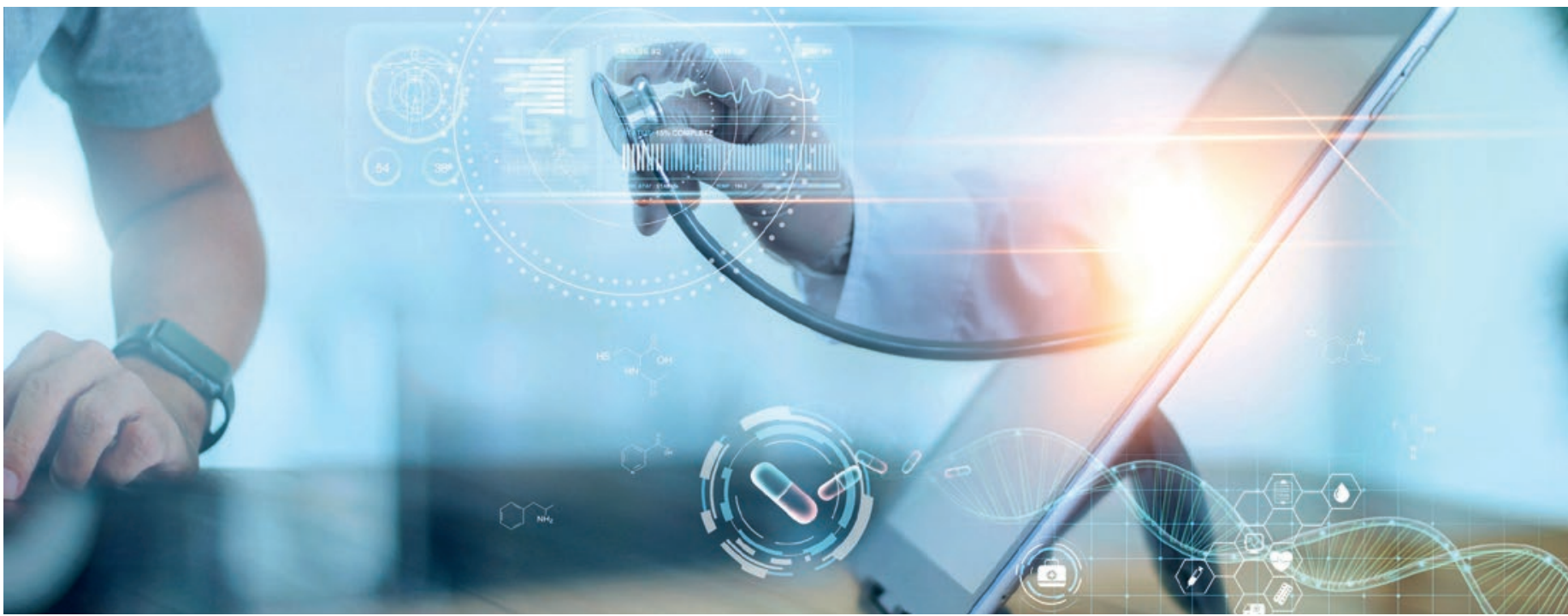

Sondage de la FMH sur les applications numériques pendant le traitement

\title{
Digital Trends Survey 2021
}

\section{Verena Pfeiffer ${ }^{a}$, Reinhold Sojer}

a Dre rer. nat., collaboratrice scientifique, division Numérisation/eHealth de la FMH;

${ }^{b}$ Dr rer. biol. hum., chef de la division Numérisation/eHealth de la FMH

A l'heure actuelle, nous sommes encore bien loin de traiter des patient-e-s au moyen d'ordinateurs. Néanmoins, le corps médical est aujourd'hui confronté à la question de savoir quel rôle les technologies numériques peuvent et devraient jouer dans le domaine de la santé. Les médecins devront développer la capacité de distinguer les déclarations médicales numériques correctes parmi les incorrectes.

Personne, ou presque, ne remet actuellement en question l'utilisation des applications numériques de santé. A l'instar de l'aviation, et en particulier dans les domaines critiques, les services de santé ont systématiquement amélioré les systèmes en usage au cours des dernières décennies. On peut difficilement s'imaginer la médecine moderne sans ces applications, qui soutiennent notamment l'assurance qualité [1].

Les systèmes d'information utilisés à l'origine dans les hôpitaux à des fins de facturation ont été constamment développés au cours des dernières décennies, et leur utilisation s'est étendue au domaine clinique [2]. Grâce à cette évolution, on dispose de plus en plus souvent de dossiers médicaux sous forme électronique, et on peut alors utiliser les données qu'ils contiennent pour la prise de décision clinique assistée par ordinateur. Ces dernières années, les applications commerciales de santé numérique ont vu le jour à partir de programmes développés principalement dans le milieu universitaire. Elles s'étendent désormais à l'ensemble du parcours du patient (prévention, triage, diagnostic, traitement et suivi) [3] et les médecins, mais également les patientes et les patients, les uti- lisent de plus en plus. Des applis aident notamment les patient-e-s à identifier des altérations pathologiques cutanées. Les dispositifs d'auto-examen permettent des examens simples de l'oreille, de la gorge, du pharynx et de la peau grâce aux fonctions intégrées de thermomètre, stéthoscope, otoscope et caméra [4]. La Suisse connaît actuellement un élan nouveau dans la diffusion des technologies de l'information dans le domaine de la santé par l'introduction du dossier électronique du patient (DEP), grâce auquel les patient-e-s peuvent consulter les données relatives à leur traitement et les partager par voie électronique avec les professionnel-le-s de la santé [5].

Les offres sont donc nombreuses; reste à déterminer les avantages et les besoins. Quels avantages le corps médical et la population suisse reconnaissent-ils dans les applications numériques de santé? Celles qui sont proposées répondent-elles à leurs besoins? Quelles applications électroniques les médecins doivent-ils envisager lorsqu'ils numérisent ou conçoivent un cabinet de médecine générale? Voilà des questions auxquelles le Digital Trends Survey 2021 a cherché à fournir des réponses. 


\section{Le Digital Trends Survey 2021}

Mené par la FMH, ce sondage sur les tendances dans le domaine de la numérisation a pour objectif d'enquêter sur les besoins et les avantages des nouvelles applications numériques de santé, d'après la perception subjective du corps médical et de la population. Il s'oriente selon le concept du parcours du patient (patient journey) qui englobe tous les événements vécus par la patiente ou par le patient, que ce soit avant, pendant et après une consultation médicale. Le Digital Trends Survey 2019 s'était intéressé aux applications numériques «avant le traitement». Cette année, le sondage a abordé leur utilisation "pendant le traitement», ainsi que l'influence de la pandémie de Covid-19 sur la numérisation [6].

\section{Méthode}

Un sondage en ligne a été réalisé d'octobre à novembre 2020, auprès de 507 médecins travaillant dans le secteur ambulatoire et 2096 personnes résidant en Suisse ( $\geq 18$ ans). La répartition par âge et par sexe des participant-e-s est présentée dans la figure 1. La méthode et le questionnaire ont été développés conjointement avec des médecins et gfs.bern. La validité du questionnaire a été vérifiée au moyen de prétests cognitif et quantitatif. Le questionnaire comprenait des questions établies avec une échelle de Likert classique, ainsi qu'une expérience de prise de décision basée sur la méthode de l'analyse conjointe. Il s'agit d'un procédé multivarié qui mesure l'évaluation globale d'un produit au moyen d'une compilation de différentes propriétés. Pour cela, on mesure quelle propriété se révèle particulièrement importante dans cette évaluation. Pour l'expérience de prise de décision, les cinq dimensions suivantes ont été analysées: processus administratifs, systèmes d'appel, personnel spécialisé, examen et traitement. Les personnes interrogées étaient priées de choisir l'un des deux cabinets médicaux hypothétiques dont les différentes dimensions présentaient un degré de numérisation (analogique, partiellement numérique, entièrement numérique) sélectionné au hasard. Les médecins devaient s'imaginer pla-

Démographie

\begin{tabular}{lll} 
& Population & Corps médical \\
\hline Sexe & Hommes: $49,2 \%$ & Hommes: $53,8 \%$ \\
& Femmes: $50,7 \%$ & Femmes: $46,2 \%$ \\
& Sans réponse: $0,1 \%$ & Sans réponse: $0 \%$ \\
\hline Répartition par âge & $18-39: 35 \%$ & $30-39: 6,7 \%$ \\
& $40-64: 42,4 \%$ & $40-49: 27 \%$ \\
& $\geq 65: 22,1 \%$ & $50-59: 37,9 \%$ \\
& Sans réponse: $0,5 \%$ & $60-69: 28,4 \%$ \\
& & Sans réponse: $0 \%$ \\
\hline
\end{tabular}

Figure 1: Répartition des échantillons par âge et par sexe. nifier un nouveau cabinet médical et indiquer quelle combinaison d'offres plus ou moins numériques des deux cabinets médicaux était la plus intéressante à leur avis. La population devait désigner le cabinet qu'elle choisirait pour un traitement.

\section{Résultats du sondage}

Les résultats du Digital Trends Survey 2021 montrent que toutes les personnes interrogées considèrent l'utilisation du numérique dans le domaine de la santé comme importante. Deux tiers des médecins travaillant dans le secteur ambulatoire conservent leurs dossiers médicaux sous forme électronique dans le système d'information primaire de leur cabinet. La population y voit un avantage évident. A la question de l'optimisation du recours aux possibilités numériques dans le système de santé, les réponses diffèrent par rapport à celles de 2019. En effet, 25\% des médecins interrogés estiment qu'ils exploitent pleinement le potentiel actuel des soins numériques, alors qu'ils étaient $64 \%$ en 2019. La pandémie de Covid-19 pourrait avoir occasionné ce changement d'opinion. Malgré l'utilisation de dossiers médicaux électroniques dans les cabinets, l'échange de données relatives à la pandémie est associé à des tâches administratives importantes et entraîne souvent une comptabilité à double entrée, dont les coûts ne sont pas couverts.

\section{La pandémie de Covid-19 incite à améliorer la qualité et la disponibilité des données numériques pertinentes}

La pandémie de Covid-19 a mis en lumière les difficultés rencontrées en Suisse dans l'échange de données entre les différents acteurs (professionnel-le-s de la santé, autorités, etc.). Toutes les personnes interrogées estiment qu'il est nécessaire d'agir dans ce domaine. La proportion de la population qui considère cette nécessité d'agir comme pertinente est supérieure de $10 \%$ à celle du corps médical (37 resp. 27\%). Il faut améliorer la qualité et la disponibilité des données numériques relatives à la pandémie de Covid-19. La compréhension scientifique et la gestion efficace de la pandémie reposent sur l'évaluation rapide et complète de ces données au niveau national.

\section{L'expérience de prise de décision montre que les applications numériques qui remplacent complètement les prestations médicales de base suscitent peu d'intérêt}

L'objectif de l'expérience de prise de décision était de déterminer quels avantages les médecins travaillant 

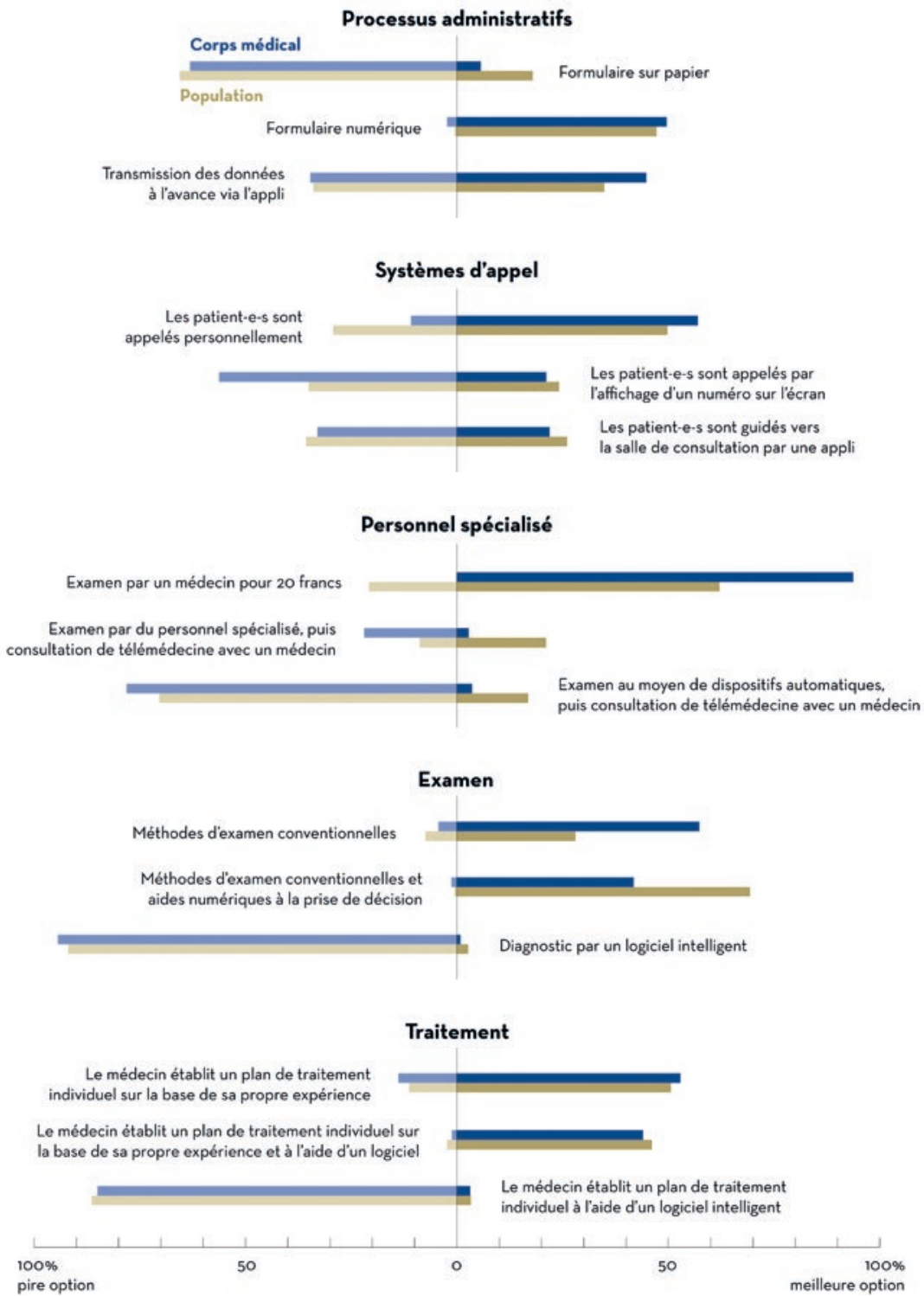

Figure 2: Evaluation des prestations d'un cabinet médical en fonction de leur degré de numérisation. Plus les barres tendent vers la droite ou vers la gauche, plus l'utilisation d'une prestation est considérée comme une bonne ou une mauvaise option.

dans le secteur ambulatoire et la population attribuent aux prestations d'un cabinet médical en fonction de leur degré de numérisation (fig. 2).

Les personnes interrogées (corps médical 50\%, population 47\%) apprécient l'option où les patient-e-s saisissent les données administratives sous forme numérique à l'aide d'une tablette fournie par le cabinet médical; à l'inverse de l'enregistrement de ces données sur papier, qui ne présente aucun avantage $(63 \%$ du corps médical et $65 \%$ de la population n'y voient aucun intérêt). L'appel personnel classique des patient-e-s en salle d'attente constitue l'option d'appel préférée (corps médical 57\%, population 50\%). Toutes les personnes interrogées considèrent l'examen physique effectué personnellement par le médecin comme l'option la meil- leure (corps médical 94\%, population 62\%), même préférable aux options numériques. L'examen effectué au moyen d'un dispositif automatisé et suivi d'une consultation de télémédecine est une option clairement rejetée (corps médical 78\%, population 70\%). La majorité du corps médical est favorable aux méthodes d'examen conventionnelles (57\%), tandis que la population préfère que les méthodes d'examen conventionnelles soient soutenues par des aides numériques à la décision (69\%). Toutes les personnes interrogées rejettent néanmoins le diagnostic exclusivement établi par un logiciel intelligent (corps médical 94\%, population $92 \%)$. Il en va de même, en matière de traitement, pour l'élaboration automatisée d'un plan thérapeutique par un logiciel intelligent (85\% du corps médical et $86 \%$ de la population rejettent cette option). Tandis qu'une grande proportion des personnes interrogées préfèrent que le médecin conçoive le plan de traitement sur la base de sa propre expérience (corps médical 53\%, population 51\%), elles sont presque autant à considérer que l'aide apportée au médecin par un tel logiciel s'avère néanmoins utile pour élaborer un plan de traitement (corps médical 44\%, population 46\%). Dans l'ensemble, la population se montre un peu plus ouverte que le corps médical vis-à-vis des solutions hybrides, telles que l'examen physique soutenu par des aides numériques pour la prise de décision ou l'élaboration de plans de traitement par un logiciel intelligent.

\section{Le DEP suscite de l'intérêt}

Dans le domaine de la documentation et de l'échange de données, l'ouverture de la population à la numérisation se reflète également dans la volonté d'ouvrir un DEP, pour lequel la majorité de la population (55\%) se montre toujours intéressée, bien que l'intérêt ait diminué de 6\% par rapport à 2019. Un peu moins de la moitié de la population interrogée $(47 \%)$ ne changerait pas de médecin si celui-ci ne proposait pas de DEP. Cette raison pourrait toutefois motiver un changement définitif de cabinet médical chez une personne interrogée sur cinq. Ceci représente une augmentation de 5\% par rapport à 2019 . Cependant, la majorité de la population (84\%) s'estime très satisfaite du service fourni par les médecins de famille. Quant aux offres numériques proposées par les cabinets médicaux, la satisfaction des patient-e-s a même légèrement augmenté (41\%) par rapport à 2019 (31\%). Toutefois, parmi la population, près d'un quart (24\%) des personnes interrogées s'estiment plutôt ou très insatisfaites des offres numériques que proposent les médecins de famille. Dès lors, un potentiel de développement et d'expansion subsiste encore dans ce domaine. 


\section{Discussion et perspectives}

Les résultats du sondage apportent des réponses aux questions posées en introduction sur les avantages et les besoins en matière de numérisation. Le corps médical envisage les offres numériques pour simplifier les processus administratifs. Disposer de plus de temps pour les traitements médicaux personnels représente également un avantage aux yeux de la population. Les applications numériques de santé qui remplacent les prestations médicales de base semblent toutefois passer à côté de l'essentiel. Ainsi, toutes les personnes interrogées rejettent l'auto-examen au moyen d'un dispositif automatisé et la prise de la décision thérapeutique par un logiciel intelligent. Les approches hybrides, qui consistent à combiner des méthodes conventionnelles avec des aides numériques à la prise de décision, suscitent toutefois de l'intérêt auprès de la population. Si le choix est fait de numériser un cabinet, il est alors souhaitable d'utiliser des applications permettant de réduire les tâches administratives, par exemple avec la saisie des données dans un formulaire numérique directement par les patient-e-s tout en maintenant le contact personnel, tel que l'appel classique en salle d'attente, car son importance est reconnue par les personnes interrogées, au même titre que l'examen physique effectué personnellement par le médecin. Les patient-e-s peuvent cependant tout à fait envisager des solutions hybrides, telles que l'élaboration de plans thérapeutiques au moyen d'un logiciel intelligent. Par ailleurs, il convient de tenir compte du raccordement au DEP, qui devra permettre l'échange de données relatives au traitement entre professionnel-le-s de la santé. Une personne interrogée sur cinq changerait de cabinet médical si le DEP ne faisait pas partie des prestations proposées. Il est donc nécessaire que les médecins de famille jouent un rôle plus actif dans le développement du DEP et fassent entendre leurs revendications en faveur d'un DEP véritablement utile. Dans le contexte de la pandémie de Covid-19, le DEP présenterait certainement des avantages. En effet, l'enregistrement précis des nouvelles infections en Suisse ne s'est pas déroulé sans certaines difficultés, ce qui peut être attribué à des infrastructures de données sous-optimales [7]. Tant que l'introduction du DEP à l'échelle nationale n'est pas aboutie, ces données ne peuvent pas être simplement utilisées dans les systèmes secondaires. En règle générale, les systèmes d'information sanitaire, à savoir la collecte de données coordonnée et habituelle, aussi entre l'ambulatoire et l'hospitalier,

Division Numérisation/

eHealth de la FMH

ehealth[at]fmh.ch ainsi que les flux de communication automatisés entre les différents acteurs (professionnel-le-s de la santé, autorités, etc.), devraient être mis en place avant une pandémie, et non en cours de route. Cela garantirait que celles et ceux qui fournissent les données soient déjà familiarisés avec les outils et les canaux de notification. En cas de pandémie, on pourrait alors disposer de ces données nationales et les utiliser pour affiner les mesures de protection [8].

Certains pays européens profitent déjà des avantages d'applications numériques de santé depuis des décennies. La Finlande dispose d'un dossier médical électronique depuis vingt ans et les ordonnances y sont délivrées sous forme numérique depuis plus de dix ans [9]. En Estonie, la loi exige depuis plus d'une décennie l'utilisation de l'ordonnance électronique et du dossier électronique du patient [10].

A chaque pays de définir le sens et la finalité qu'il entend accorder à la numérisation. Les résultats du Digital Trends Survey 2021 contribuent à mettre en évidence les souhaits et les limites que posent les médecins quant au cadre à lui donner dans notre pays. Au final, la numérisation aurait le potentiel de soutenir la transition du curatif vers le préventif dans le système de santé, de centrer l'humain au cœur des soins, de promouvoir l'autonomie des patient-e-s et de rendre le système de santé plus efficace et plus sûr [11].

\section{Références}

1 Middleton B, Bloomrosen M, Dente MA, Hashmat B, Koppel R, Overhage JM, et al. Enhancing patient safety and quality of care by improving the usability of electronic health record systems: recommendations from AMIA. J Am Med Inform Assoc. 2013;20(e1): e2-8.

2 Evans RS. Electronic Health Records: Then, Now, and in the Future. Yearb Med Inform. 2016 May 20;Suppl 1:S48-61.

3 Robinson A, Slight RD, Husband AK, Slight SP. Designing the Optimal Digital Health Intervention for Patients' Use Before and After Elective Orthopedic Surgery: Qualitative Study. J Med Internet Res. 2021 Mar 8;23(3):e25885.

4 Don DM, Koempel JA, Fisher LM, Wee CP, Osterbauer B. Prospective Evaluation of a Smartphone Otoscope for Home Tympanostomy Tube Surveillance: A Pilot Study. Ann Otol Rhinol Laryngol. 2021 Feb;130(2):125-32.

5 Loi fédérale du 19 juin 2015 sur le dossier électronique du patient (LDEP, RS 816.1).

6 Digital Trends Survey 2019. https://www.fmh.ch/fr/themes/ ehealth/tendances-et-technologies.cfm

7 Horgan D, Hackett J, Westphalen CB, Kalra D, Richer E, Romao M, et al. Digitalisation and COVID-19: The Perfect Storm. Biomed Hub. 2020 Sep-Dec;5(3):1341-63.

8 Schmidt AE, Abboud LA, Bogaert P. Making the case for strong health information systems during a pandemic and beyond. Arch Public Health. 2021;79:13.

9 Schmitt-Sausen N. Finnland lebt die Digitalisierung. Dtsch Arztebl. 2018;115(41):A-1802/B-1516/C-1502.

10 Thiel R, Deimel L, Schmidtmann D, Piesche K, Hüsing T, Rennoch J, et al. \#SmartHealthSystems: Auszug Estland. Bertelsmann Stiftung; 2018 [Internet]. https://www.bertelsmann-stiftung.de/de/ publikationen/publikation/did/smarthealthsystems/

11 Odone A, Buttigieg S, Ricciardi W, Azzopardi-Muscat N, Staines A Public health digitalization in Europe. Eur J Public Health. 2019 Oct;29(Suppl 3):28-35. 